\title{
Neuronal cell-type and circuit basis for visual predictive processing
}

Jinmao Zou ${ }^{1,2+}$, Lawrence Huang ${ }^{3+}$, Lizhao Wang ${ }^{4}$, Yuanyuan $\mathrm{Xu}^{1,2}$, Chenchang $\mathrm{Li}^{1,2}$, Qilin Peng ${ }^{1,2}$, Hongkui Zeng ${ }^{3}$, Siyu Zhang ${ }^{4^{*}}, \mathrm{Lu} \mathrm{Li}^{1,2,5^{*}}$

\section{Affiliations:}

1. Medical Research Centre, Sun Yat-sen Memorial Hospital

Sun Yat-sen University

107 Riverside West Road

Guangzhou, Guangdong Prov., China 510120

2. Guangdong Provincial Key Laboratory of Malignant Tumor Epigenetics and Gene Regulation,

Sun Yat-sen Memorial Hospital

Sun Yat-sen University

107 Riverside West Road

Guangzhou, Guangdong Prov., China 510120

3. Allen Institute for Brain Science

615 Westlake Ave N

Seattle, WA 98109

4. Center for Brain Science of Shanghai Children's Medical Center, Department of Anatomy and Physiology, Shanghai Jiao Tong University School of Medicine, Shanghai 200025, China

5. Centre for Brain Science and Brain-Inspired Intelligence,

Guangdong-Hong Kong-Macao Greater Bay Area, China

† These authors contributed equally

*Correspondence should be addressed to: (L.L.) lilu67@mail.sysu.edu.cn or (S.Y.Z) zhang siyu@sjtu.edu.cn

One Sentence Summary: VIP-SST-Pyr microcircuit in visual predictive processing during voluntary running 


\section{Abstract (155 words)}

Bayesian Brain theory suggests brain utilises predictive processing framework to interpret the noisy world $d^{1-11}$.

Predictive processing is essential to perception, action, cognition and psychiatric disease ${ }^{12}$, but underlying neural circuit mechanisms remain undelineated. Here we show the neuronal cell-type and circuit basis for visual predictive processing in awake, head-fixed mice during self-initiated running. Preceding running, vasoactive intestinal peptide (VIP)-expressing inhibitory interneurons (INs) in primary visual cortex (V1) are robustly activated in absence of structured visual stimuli. This pre-running activation is mediated via distal top-down projections from frontal, parietal and retrosplenial areas known for motion planning, but not local excitatory inputs associated with the bottom-up pathway. Somatostatin (SST) INs show pre-running suppression and postrunning activation, indicating a VIP-SST motif. Differential VIP-SST peri-running dynamics anisotropically suppress neighbouring pyramidal (Pyr) neurons, preadapting Pyr neurons to the incoming running. Our data delineate key neuron types and circuit elements of predictive processing brain employs in action and perception. 


\section{Main}

To make probabilistic inference of the changing environment, brain may employ a hierarchical generative framework called predictive processing ${ }^{1-11}$. In this framework, an internal model (the upper layer) generates an 'initial guess' or prediction; the lower layer reports up the prediction-error, which is the discrepancy between the prediction and reality so the internal model can make a better guess. By repetitions, predictive processing minimises the prediction-error, leading to successful inference of the real cause so that perception or cognition may arise. From Bayesian perspectives, it lays a foundation for unifying perception, action and cognition, and accordingly, less optimized predictive processing may account for psychiatric disorders ${ }^{12}$. However, how the brain neurobiologically implements such a mathematically convincing framework remains largely unknown.

In motor tasks, frontal, parietal and retrosplenial brain regions fire preparatory neural activities preceding the action initiation, which could be neural correlates of predictive processing ${ }^{13-20}$. Recent studies have reported prediction-error encoding neurons in visual, auditory and somatosensory cortices ${ }^{21-24}$, but understandings of the circuit mechanisms remain elusive. Firstly, neural computations are carried out by brain circuits consisting of interconnected neuronal cell-types, i.e. distinct groups of neurons categorised by characteristic morphology, physiology, connectivity and genetics ${ }^{25-28}$, however, key neuron types in predictive processing are undefined. Secondly, predictive processing, by its mathematical definition, requires interplay between bottom-up and topdown processes ${ }^{1,8,11,29,30}$, yet underlying circuit elements are less delineated. Finally, predictive processing requires neuronal dynamics of both excitatory pyramidal (Pyr) cells and INs, but functional data of neuron populations that elucidate neuronal activity dynamics at single-neuron resolution are largely absent.

We investigated the neuronal cell-type and circuit basis for predictive processing in awake, head-fixed mice during brief, sporadic self-initiated running (Fig. 1a and Supplementary Fig. 1, also see Methods). Self-initiated actions employ predictive processing to suppress self-induced sensory outcomes ${ }^{2,4,8}$ and are essential to human perceptual, cognitive and social experiences, making it an ideal behavioural paradigm to dissect neural circuits of predictive processing in experimental animals. Without oversimplification, we propose that internal models in cortical motion-planning centres predict visual outcomes of running; the predictions, likely encoded by preparatory neural activities in frontal, parietal or retrosplenial brain regions ${ }^{13-20}$, are sent to V1 via top-down projections to suppress running-induced visual responses ${ }^{31}$ thus result in observable pre-running neuronal signature in V1; prediction-error encoding neurons in V1 then sent back prediction-errors through reciprocal connections between $\mathrm{V} 1$ and motion-planning areas ${ }^{23,31}$. Based on this working model, we utilised transgenic mouse lines $^{32,33}$ that cell-type specifically expressed genetically encoded Calcium (Ca) indicators ${ }^{34}$ (GECls, e.g. GCaMP6s) to identify key neuron types in predictive processing. To reveal the pre-running neuronal signature, we performed in vivo two-photon (2-p) Ca imaging of V1 layer (L) 2/3 genetically-defined neuron populations ${ }^{35}$. 
We chose to first focus on V1 L2/3 VIP INs due to their extensive innervations by top-down projections ${ }^{36}$ and importance demonstrated in predictive processing-associated cognitive functions such as learning and attention $^{37-41}$.

\section{VIP INs are robustly activated prior to self-initiated running in absence of structured visual stimuli}

We imaged 335 L2/3 VIP INs with $400 \times 400 \mu \mathrm{m}$ fields of view (FOV, $n=22$, Fig. 1b) in naïve VIP-Cre;Ai162 (GCaMP6s) mice. Mice were free to run at arbitrary times relative to the imaging start during 200 sec imaging sessions $^{42}$ (Supplementary Fig. 1). To identify neural correlates of predictive processing, we quantified perirunning (-10 to $5 \mathrm{sec}$ from running onset, Fig. 1c) fluorescence changes $(\Delta F / F)$ and constructed Peri-Running Time Histograms (PRTHs) for individual fluorescently active regions of interest (ROIs, $n=318$ ). As shown by the example FOV in Fig. 1b, VIP INs significantly increases $\Delta F / F$ during running (Supplementary Fig. 2), consistent with the activity augmentation reported during locomotion ${ }^{42}$. However, 46\% (6/13) of active ROls significantly increases $\Delta F / F$ before the running onset (latency: $-0.192 \pm 0.043 \mathrm{sec}$, mean \pm s.e.m, $n=6$, Fig. $1 \mathrm{~d}$ ), whereas in the rest 38\% (5/13) of ROIs, significant $\Delta F / F$ increase occurs after running starts (latency: $0.486 \pm 0.381 \mathrm{sec}, \mathrm{n}=5$, Fig. 1e \& Supplementary Fig. 2). For convenience, these neurons are termed VIP Pre and VIP $P_{\text {post, }}$, respectively. At population level, VIP Pre INs (36.2 $\pm 4.3 \%$, total: 121/318) distribute extensively across all 22 FOVs (Fig. 1g) with an average latency at $-0.365 \pm 0.033 \mathrm{sec}(n=121$, Fig. 1h). The fast rise of pre-running activity in mean PRTH (Fig. 1i) suggests VIP Pre receive strong and synchronous excitatory inputs prior to running initiation, e.g. the top-down preparatory neural activities. VIP Post INs, which consist of $44.6 \pm 4.1 \%$ (total: $139 / 318$ ) of the entire VIP population, show an average latency of $1.087 \pm 0.068 \mathrm{sec}(\mathrm{n}=139)$ with a slow up-ramping PRTH (Fig. $1 \mathrm{~g}$-i). In the rest $17.1 \pm 4.1 \%$ ( $n=58 / 318$ ) of VIP INs, activities are slightly suppressed (Supplementary Fig. 3). Consistent with previous studies ${ }^{42,43}$, both VIP Pre and VIP Post show similar sustained activity elevation after running start (Supplementary Figs. 3-5), suggesting common sets of excitatory sources responsible for post-running activation in both VIP Pre and VIP Post $I$ INs. This allows us to estimate the 'net' pre-running VIP activation by subtracting the average activity of VIP Post $_{\text {from that of VIP }}$ INs, which reveals a sharp, transient pre-running VIP activity (Fig. 1i) mimicking preparatory neural activities (e.g. the Readiness Potential) reported in human, monkeys and rodents ${ }^{13-20}$. Pre-running activation of VIP INs is NOT an evoked response to uncontrolled visual input, because robust, even slightly stronger pre-running VIP activation is found in mice running in darkness (112 ROIs, 6 FOVs in 3 VIP-Cre;Ai162 mice, Supplementary Figs. 4\&5). Different temporal activations of co-existing VIP Pre and VIP Post $I N s$ differentiate our findings from continuous locomotion, in which activity augments are global, non-specific and depend on neuromodulator-mediated arousal/brain state changes ${ }^{42,43}$. 
In our behavioural paradigm, running occurs randomly (Supplementary Fig. 1). This randomness validates running is self-initiated thus adequately recruits predictive processing, but makes it infeasible to deliver manipulations prior to running because now when the animal will run is unknown to the experimenter. As we show below, pre-running activation is cell-type specific to VIP INs, ruling out possibilities of artifacts by undocumented movement and/or data processing/analysis. More importantly, on average $5.5 \pm 0.85$ VIP Pre INs are activated prior to running, and 68\% (15/22) of FOVs have at least 4 VIP Pre INs identified (Supplementary Fig. 3). This clustering of VIP Pre strongly suggests common causes for VIP pre-running activation instead of stochastic fluctuations of individual VIP INs. In summary, our data demonstrate an early engagement of L2/3 VIP INs preceding self-initiated running, providing direct in vivo evidence that the VIP network plays crucial roles in visual predictive processing.

\section{Distal top-down projections provide dominant excitatory drive to pre-running VIP activation}

V1 VIP INs receive top-down, bottom-up and local recurrent excitatory inputs ${ }^{36-40}$. To delineate the interplay between top-down and bottom-up pathways pertaining to pre-running VIP activation, we examined the peri-running activities of V1 local L2/3 Pyr neurons ( $n=378$ ROIs in 9 FOVs, Fig. 2 \& Supplementary Figs. 6\&7) in Emx1-IRES-Cre;CaMK2a-tTA;Ai94 mice (expressing GCaMP6s in pan-excitatory cortical neurons). Here L2/3 Pyr activities act as the readout of bottom-up and local excitatory influences on VIP INs, because LGN and L4 bottom-up pathways provide the driving input to L2/3 Pyr neurons. Furthermore, if pre-running VIP activation is indeed part of visual predictive processing, characteristic neural dynamics, especially Pyr activity suppression, should be observed as predicted by our working model. Our data confirm this projection. As exemplified in Fig. 2a-d, contrary to VIP, only a tiny portion of Pyr neurons (2\%, 1/47 ROIs) are Pyr Pre cells (Fig. 2b); 43\% (20/47) and

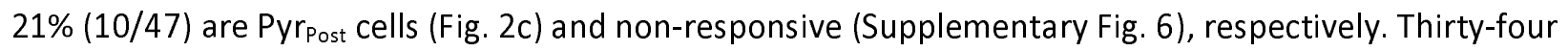
percent (16/47) of Pyr neurons, as expected, show pre-running suppression ( $\mathrm{Pyr}_{\text {sup }}$, Fig. 2d). Across all three

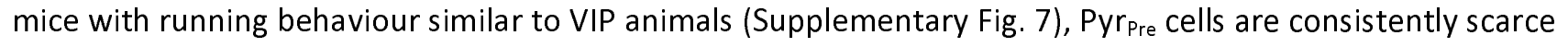
$(1.9 \pm 0.8 \%$, median: 0 , total: $8 / 378$, Fig. $2 \mathrm{e} \& \mathrm{f})$, indicating the pre-running activation is an intrinsic property of VIP INs. The scarcity of Pyr Pre $_{\text {re }}$ neurons suggests bottom-up and local pathways provided little, if any, excitatory drive to pre-running VIP activation. Instead, the fact that the majority of Pyr neurons $(64.9 \pm 6.8 \%$, median: 66.7\%, total: 252/378) are Pyr Post $_{\text {c }}$ cells indicate local Pyr activities contribute more to post-running VIP activation. These results are consistent with previous studies showing LGN and L4 only slightly increase activity during, but not before locomotion ${ }^{43,44}$. Importantly, in $30.9 \pm 6.6 \%$ (median: $32.0 \%$, total: 118/378) of Pyr neurons, we find profound pre-running suppression (Fig. 2h \& Supplementary Fig. 7), providing experimental support that the observed peri-running dynamics of Pyr neurons are recruited by visual predictive processing in voluntary running. 
Taken together, these data demonstrate bottom-up/local excitatory inputs are insufficient to drive the prerunning activation of VIP INs.

To further determine the excitatory source of pre-running VIP activation, we conducted Cre-dependent retrograde mono-synaptic tracing of distal inputs to V1 VIP INs with engineered rabies virus in VIP-IRES-Cre;Ai14 mice. Unilateral viral injections were made at superficial locations of $\mathrm{V} 1$, and we quantified ipsilateral neocortical input density to V1 VIP INs. Our tracing results confirm V1 L2/3 VIP INs are directly innervated by distal monosynaptic excitatory top-down projections from ipsilateral motion-planning cortical regions ${ }^{36}$ (Fig. $2 \mathrm{i} \& \mathrm{j})$. Fluorescent positive presynaptic cells had typical morphology of projection neurons and distribute mostly in deep cortical layers (Fig. 2i). Areal distribution is widespread but biased, with more than $85 \%$ inputs originated from lateral posterior parietal cortex (PTLp), retrosplenial (RP), and somatosensory (SS) cortices (Fig. 2j), which are cortical areas known responsible for motion-related preparatory neural activities in rodents ${ }^{13-20}$. These monosynaptic excitatory top-down projections, which convey predictions by the 'upper layer', provide the anatomical substrate required by the fast, time-locked pre-running activation of VIP INs we observe in V1.

\section{VIP-SST motif is essential to visual predicative processing}

In mouse V1, INs consist of three complete, non-overlapping types, namely Parvalbumin (PV)-, SST- and

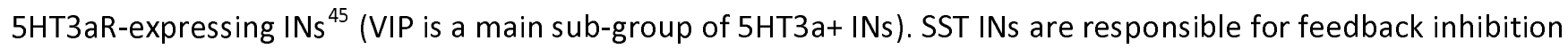
in bottom-up information processing and important to learning, attention and orientation selectivity ${ }^{37-42 E r r o r !}$ Bookmark not defined. Meanwhile, SST INS are the primary post-synaptic target of neighboring VIP INs ${ }^{37-42,46}$, and we have previously discovered that two subpopulations of V1 L2/3 SST INs are closely associated with the bottomup and top-down pathway, respectively ${ }^{35}$. These results indicate SST INs may play crucial roles in top-down and bottom-up crosstalk of predictive processing. To further dissect circuit mechanisms for visual predictive processing, we imaged and analyzed peri-running activities of L2/3 SST INs ( $n=304$ ROls in 23 FOVs) using SSTIRES-Cre;Ai163 (GCaMP6s) mice. Consistent with published data ${ }^{35,41,42}$, SST INs are highly spontaneously active in awake V1 (Fig. 3a). However, unlike VIP INs, much fewer SST INs are activated prior to running initiation (Fig. 3b \& c). On average only $5.8 \pm 1.9 \%$ of SST INs (median: 0 , total: $17 / 284$ ) are SST pre cells (Fig. $3 d-f$, Supplementary Figs. 8\&9), with all showing post-running activity overwhelms that of pre-running (Fig. 3f). This de facto weak pre-running SST activity, which is in sharp contrast with VIP INs, dismisses VIP Pre pre-running activation is caused by coincidental spontaneous firing or undocumented movement. It further suggests preparatory neural activities via the top-down pathway have relatively little contribution to overall SST activation. About $67.1 \pm 4.7 \%$ (median: 63.6\%, total: $186 / 284$ ) and $27.1 \pm 4.6 \%$ (median: $25 \%$, total: $81 / 284$ ) of SST INs show post-running activation

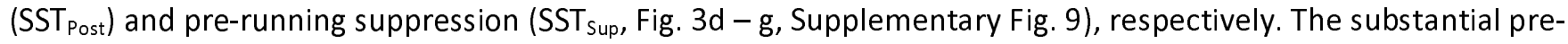


running SST suppression confirms SST INs are the downstream target of pre-running activated VIP ${ }^{46}$ and supports a novel VIP-SST motif in visual predictive processing. This finding is consistent with previous studies of anatomical connectivity ${ }^{46}$ and predictive processing-associated cognitive functions such as attention and learning ${ }^{37-41}$, thus represents a circuit motif different from the SST-VIP circuit reported for the plasticity of visual mismatch signal (i.e. prediction-error) processing during development ${ }^{47}$. In our data, more than half of SST INs are SST $_{\text {post }}$ cells, which are closely associated with bottom-up and local excitatory pathways, highly consistent with our previous finding of two SST subtypes ${ }^{35}$. Functionally, pre-running SST suppression induces early disinhibition, and post-running SST activation causes delayed inhibition to neighbouring postsynaptic Pyr neurons, respectively. This spatiotemporally coordinated activation of the VIP-SST motif may result in a synergistic 'push-pull' like regulation of the responsiveness of Pyr neurons. To summarise, our results demonstrate the VIP-SST motif is a key element of neural circuits for predictive processing.

\section{Spatiotemporally coordinated peri-running dynamics in the VIP-SST-Pyr microcircuit adaptively regulate V1 visual information processing}

Psychology and cognitive neuroscience literature has long suggested predictive processing in voluntary actions filters out self-induced sensory responses to efficiently process salient sensory inputs from the periphery. This matches well with the novel peri-running neural dynamics we report, in particular the pre-running activation and suppression of VIP and Pyr neurons, respectively. Bulk manipulation of VIP/SST activity through optogenetics, as demonstrated by systems neuroscience studies ${ }^{37-41,48-50}$, sufficiently modulates orientation tuning and other response properties of neighbouring Pyr neurons. However, do the peri-running Pyr dynamics truly represent the visual information processing being adapted to the incoming running, or just some global effects non-specific to predictive processing? We reason that, if $\mathrm{Pyr}_{\text {sup }}$ neurons are effectively recruited by predictive processing associated with running, statistically they should be biased towards the expected least informative sensory features, which, like the 'negative image', ought to be filtered out. In other words, the suppression of $\mathrm{Pyr}_{\text {Sup }}$ should be anisotropic. The post-running excitation, however, should be isotropic in order to facilitate the error reporting or the detection of novel incoming stimuli currently unknown to the brain. Our data show it is the case (Fig. 4). We managed to map orientation tuning curves of $\operatorname{Pyr}_{\text {sup }}\left(n=107\right.$ ) and Pyr Post $_{\text {c }}$ cells ( $n=247,8$ FOVs, Fig. 4a\&b) we investigated during self-initiated running (see Methods). Overall Pyr responses to drifting gratings are consistent with published results, but Pyr $_{\text {sup }}$ differ significantly from Pyr $_{\text {Post }}$ cells in orientation selectivity ( $p=0.012$, Kolmogorov-Smirnov test, Fig. 4c). Pyr $r_{\text {sup }}$ are more sharply tuned (OSI: $0.613 \pm$ 0.027 vs. $0.531 \pm 0.018, p=0.012$, $t$-test, Fig. $4 c$ ) and their preferred orientations $\left(\theta_{\text {pref }}\right)$ are not uniformly distributed but strongly biased at $45^{\circ}$ and $90^{\circ}\left(p=2.7 \times 10^{-15}\right.$, Pearson's chi-squared test, Fig. $4 \mathrm{~d}$ ), supporting an 
anisotropic pre-running suppression. Pyr $r_{\text {post }}$ 's $\theta_{\text {pref, }}$, however, follows a uniform distribution $(p=0.96$, Pearson's chi-squared test, Fig. 4d), indicating an isotropic post-running excitation. To obtain further insights, we binned $\theta_{\text {pref }}$ into 4 behaviourally cardinal pairs, which is justified by the low direction selectivity of V1 Pyr cells (Supplementary Fig. 10), and quantified $\theta_{\text {pref }}$ distributions by FOVs to control possible sampling bias. Results confirm an isotropic excitation of $\mathrm{Pyr}_{\text {post }}$ neurons, as $\theta_{\text {pref }}$ of $\mathrm{Pyr}_{\text {post }}$ distributes uniformly and shows no apparent preference (Fig. 4e). This is in sharp contrast with the highly anisotropic effect in $\mathrm{Pyr}_{\text {sup }}$ cells: suppression readily suppress, but does not over-suppress $\mathrm{Pyr}_{\text {sup }}$ tuned to $0^{\circ} / 180^{\circ}(p=0.99)$ and $135^{\circ} / 315^{\circ}(p=0.57, t$-test $)$, which is consistent with its projected function in visual flow. However, suppression is stronger to $\mathrm{Pyr}_{\text {sup }}$ tuned to $90^{\circ} / 270^{\circ}(p=0.04)$, but weaker to $45^{\circ} / 225^{\circ}(p=0.01$, Fig. 4 e). Functionally this represents a re-allocation by the VIP-SST disinhibition of V1 processing power towards $45^{\circ} / 225^{\circ}$, which is behaviourally more informative to the animal because for running in the horizontal plane, an object more likely comes from top-front based on previous experience (Fig. 4f). As a result, activity tuned to $90^{\circ} / 270^{\circ}$ reduces, probably due to the limited amount of computational resource in V1.

To establish causal relationship, we optogenetically manipulated activities of top-down projections and VIP INs in anesthetised mouse V1 with channelrhodopsin (ChR2) and halorhodopsin, respectively ${ }^{39}$ (Supplementary Fig. 11, see Methods). Cell-attached recordings were conducted to compare V1 Pyr responses to drifting gratings

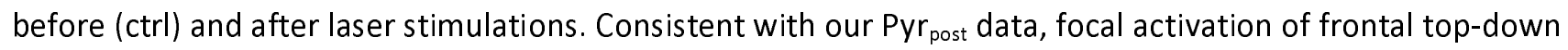
axons in $\mathrm{V} 1$ (top-down+) causes disinhibition of V1 Pyr responses ${ }^{37-42,46}$, which is completely reversed by the inactivation of VIP INs (Normalized $\theta_{\text {pref }}$ response, top-down+ vs. top-down+/VIP-, p = 0.015, Supplementary Fig. 11b). In addition, inactivating VIP INs during top-down activation, but not top-down activation alone, significantly sharpens the orientation tuning of V1 Pyr neurons (ctrl vs. top-down+/VIP-, p =0.04, Supplementary Fig. 11c). This sharpening effect validates pre-running VIP activation suppresses sharply tuned Pyr $_{\text {sup }}$ neurons, because blocking VIP activities during top-down activation would release those Pyr neurons from suppression thus result in a sharpening effect of Pyr tuning curves, which is exactly what the optogenetic data show. Functionally Pyr $_{\text {sup }}$ suppression may enhance behavioural performance ${ }^{51}$. Due to the randomness of self-initiated running, as we have pointed out, it remains infeasible to present optogenetic manipulation before running to examine the running-dependent anisotropic suppression (Fig. 4f). In summary, these results experimentally verify the causal role of the VIP-SST-Pyr microcircuit in predictive processing and provide pivot in vivo evidence for neurobiological substrate of predictive processing.

\section{Discussion}


In the present study, we have dissected the neuronal cell-type and circuit basis for predictive processing, which is a fundamental concept in cognitive neuroscience, psychology, cybernetics and artificial intelligence yet with limited systems neuroscience knowledge ${ }^{1-11,52}$. Employing a self-initiated running paradigm, we discover robust VIP activation prior to running thus reveal a novel neuronal signature of visual predictive processing in voluntary action. Preparatory activation in V1 is selective to VIP INs, suggesting the pivot role of VIP INs in topdown and bottom-up crosstalk of predictive processing. We further identify that, with converging anatomical and functional evidence, the VIP-SST-Pyr microcircuit is the key element of predictive processing due to the fact that it is the recipient of long-range top-down projections from the 'upper layer' brain regions known to fire preparatory neural activities and its unique peri-running dynamics preadapt V1 Pyr neurons to the incoming running, which matches well with theoretical projections. Together, these findings illustrate the neural substrate of predictive processing, thus bridge the long-existing gap between psychology/cognitive neuroscience and systems neuroscience.

The SST-VIP circuit has been previously suggested for visual mismatch signal (prediction-error) processing ${ }^{47}$, yet the upstream neural circuitry of predictive processing remains unknown. Our data of the VIP-SST-Pyr circuit complete this missing link and complement the SST-centred circuitry for prediction-error plasticity during development. VIP-SST motif plays critical roles in sensory information processing (e.g. gain control), selective attention and associative learning ${ }^{37-41,48-50}$. Here we present novel evidence for its function in voluntary actions. VIP-SST-Pyr microcircuit may universally underlie neural computations for predictive processing, our results thus will help to generate better understandings of the neurobiology of cognitive functions important to human nature such as sense of agency/volition ${ }^{53}$. Since the seminal work of the 'Readiness Potential' in human supplementary motor area, preparatory neural activity has been found relevant to voluntary action planning, decision-making and time sensation ${ }^{2-20}$. The identification of the VIP-SST-Pyr mirocircuit could shed new light on pinpointing neural substrate of sophisticated brain functions for more fine-grained, mechanistic knowledge. Future studies are required to elucidate genetic, molecular and connective signatures of VIP/SST subgroups to allow better-targeted manipulations of higher-order cognitive functions, in order to develop novel treatments on psychiatric disorders without undesired confounding effects ${ }^{12}$.

\section{Acknowledgements}

This work was supported by grants from National Natural Science Foundation of China NSFC 31871055 (L.L.), NSFC 31871051 (S.Y.Z), Guangdong Science and Technology Department $2017 B 030314026$ (L.L.) and 2018B030334001 (L.L.). We thank Dr. Karel Svoboda and his laboratory for technical assistance. The authors 
wish to thank the Allen Institute founders, Paul G. Allen and Jody Allen, for their vision, encouragement and support.

\section{Author Contributions}

L.L. conceived and supervised the project. L.H. and L.L. performed Ca imaging experiments. L.L. and J.Z. analyzed Ca imaging data. L.Z.W. and S.Y.Z. conducted the retrograde rabies tracing and optogenetics experiments. Y.Y.X., C.C.L. and Q.L.P. helped Ca imaging data analysis. L.L wrote the paper with inputs from all authors.

\section{Competing interests}

The authors declare no competing financial interests.

\section{Data availability}

The authors declare that all data supporting the findings of this investigation are available within the article, its Supplementary figures, and from the corresponding authors upon reasonable request. 

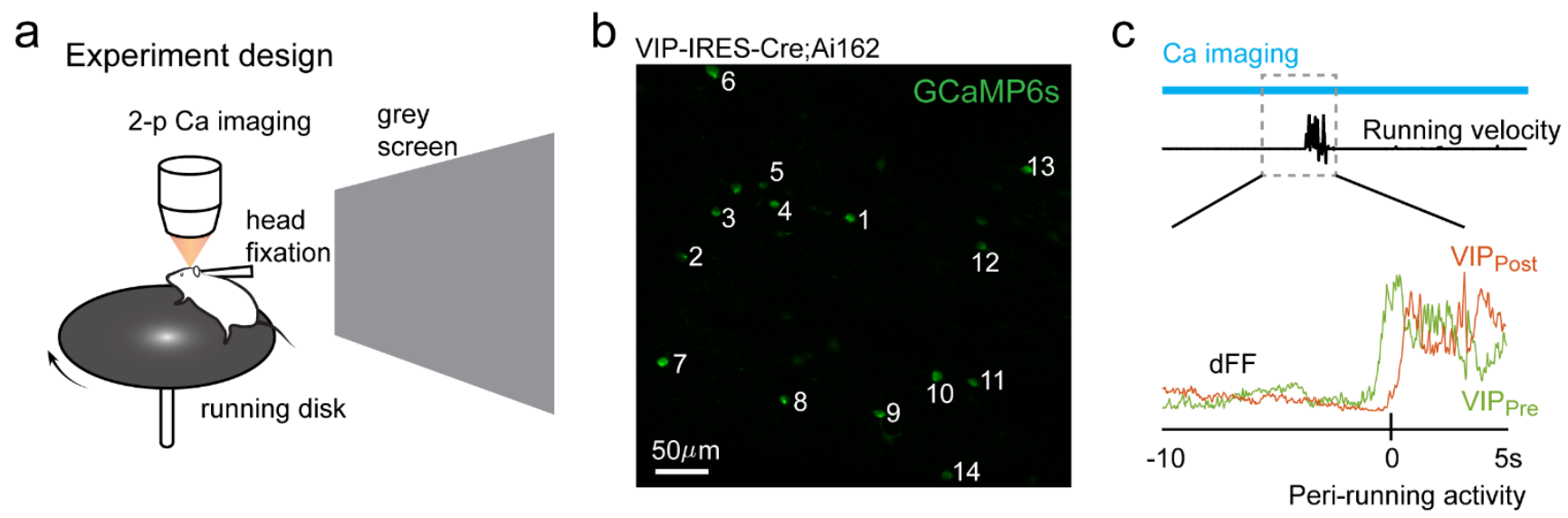

d

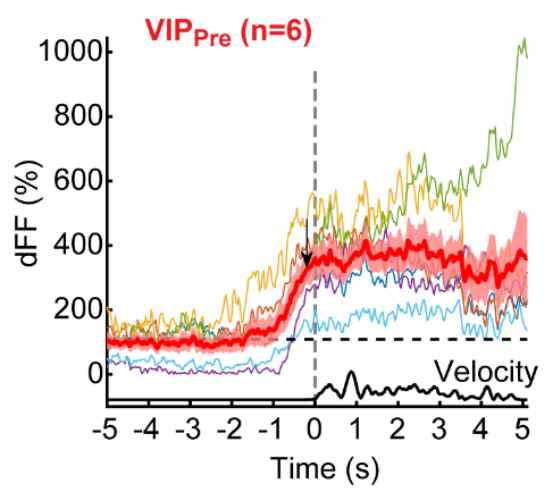

9

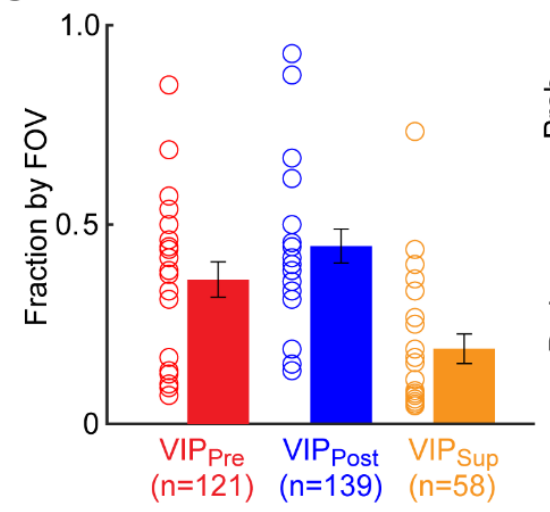

h e
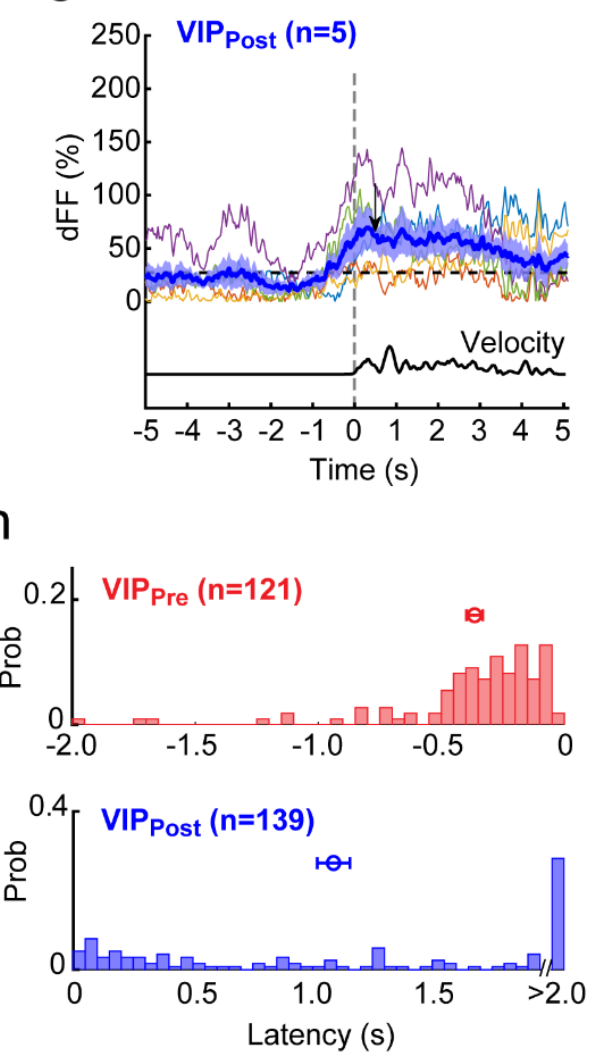

f

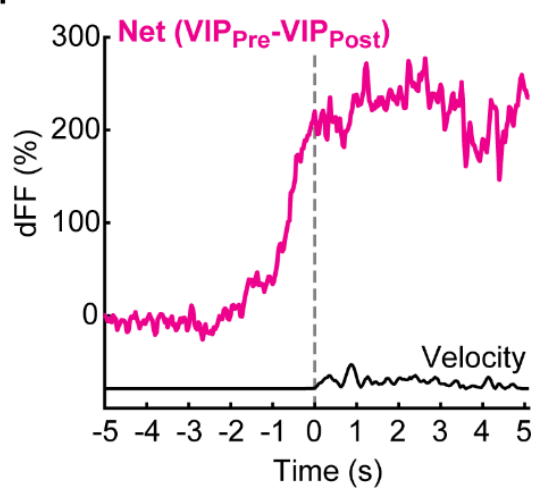

i

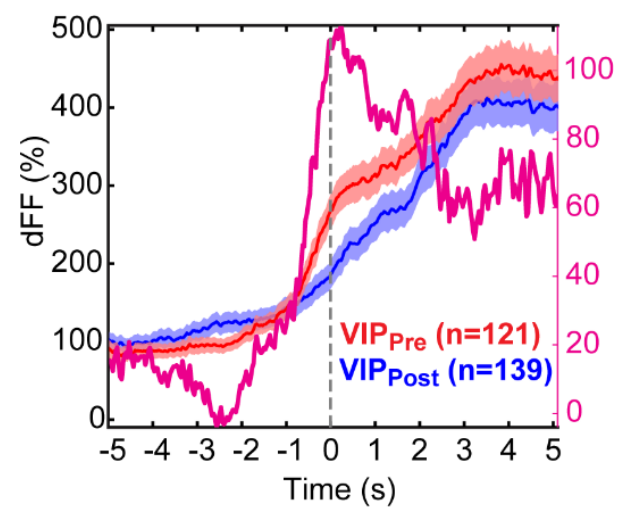


Figure 1. Robust activation of V1 VIP neurons prior to self-initiated running. a. Experimental setup showing Ca Imaging in naïve head-fixed mice during self-initiated running in absence of structured visual stimulations. $\mathbf{b}$. Example field of view (FOV, imaged at $174 \mu \mathrm{m}$ sub-pial) in a VIP-Cre;Ai162 (GCaMP6s) mouse. Numbers on the Zprojection (time series) image of the FOV indicate regions of interest (ROIs). C. Peri-running analysis of $\Delta \mathrm{F} / \mathrm{F}$ by Peri-Running Time Histograms (PRTHs). Bottom shows example VIP Pre and VIP Post neurons identified. $\mathbf{d}-\mathbf{f}$. Time courses of peri-running activities of $\operatorname{VIP}_{\text {Pre }}(\mathbf{d}), \operatorname{VIP}_{\text {Post }}$ neurons $(\mathbf{e})$ in $\mathbf{b}$ and 'Net' activation ( $\left.\mathbf{f}\right)$. Thin and thick solid lines plot individual cells and group averages, respectively. Bottom trace plots running velocity. Arrows mark mean latency. Note the substantial activity increase within $\sim 1$ s prior to running initiation. For display purposes, only $\Delta \mathrm{F} / \mathrm{F}$ within $-5 \mathrm{sec}$ to $5 \mathrm{sec}$ from the running onset is shown. Horizontal dashed line: pre-running baseline; vertical dashed line: running onset; Shade area: s.e.m. $\mathbf{g}-\mathbf{i}$. Population data showing fractions of VIP $P_{\text {Pre, }}$ VIP Post and VIP Sup neurons by FOV (g); latency distributions of VIP Pre and $\mathrm{VIP}_{\text {Post }}$ neurons $(\mathbf{h})$; average time courses of

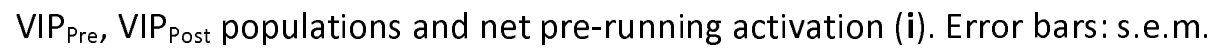


bioRxiv preprint doi: https://doi org/10.1101/2021.12 31.474667; this version posted January 2, 2022 The copyright holder for this preprint (which was not certified by peer review) is the author/funder, who has granted bioRxiv a license to display the preprint in perpetuity. It is made available under aCC-BY-NC-ND 4.0 International license.

a
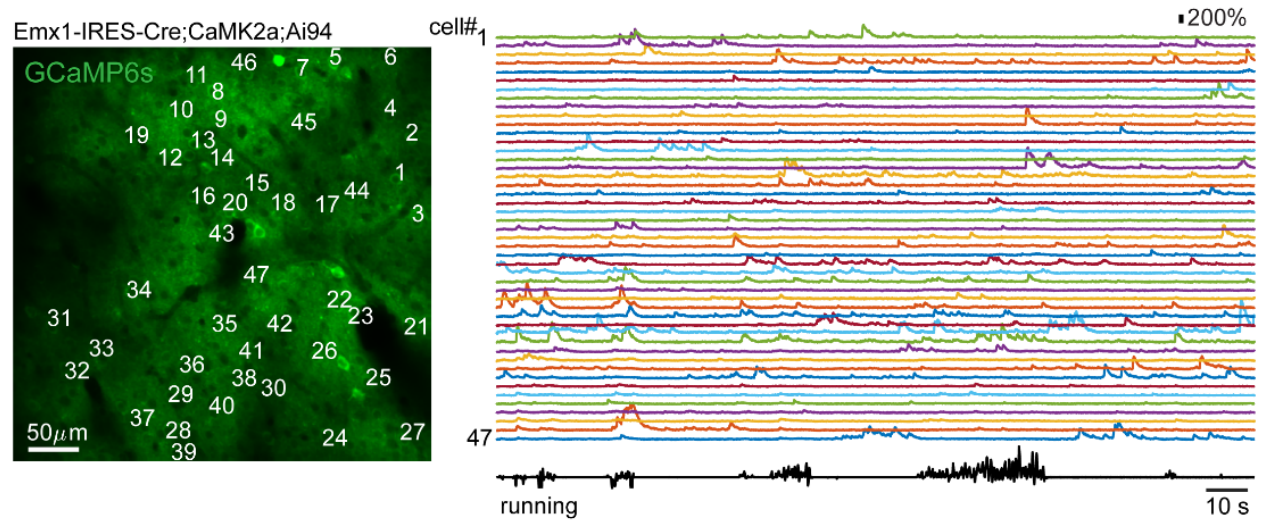

b

C

d
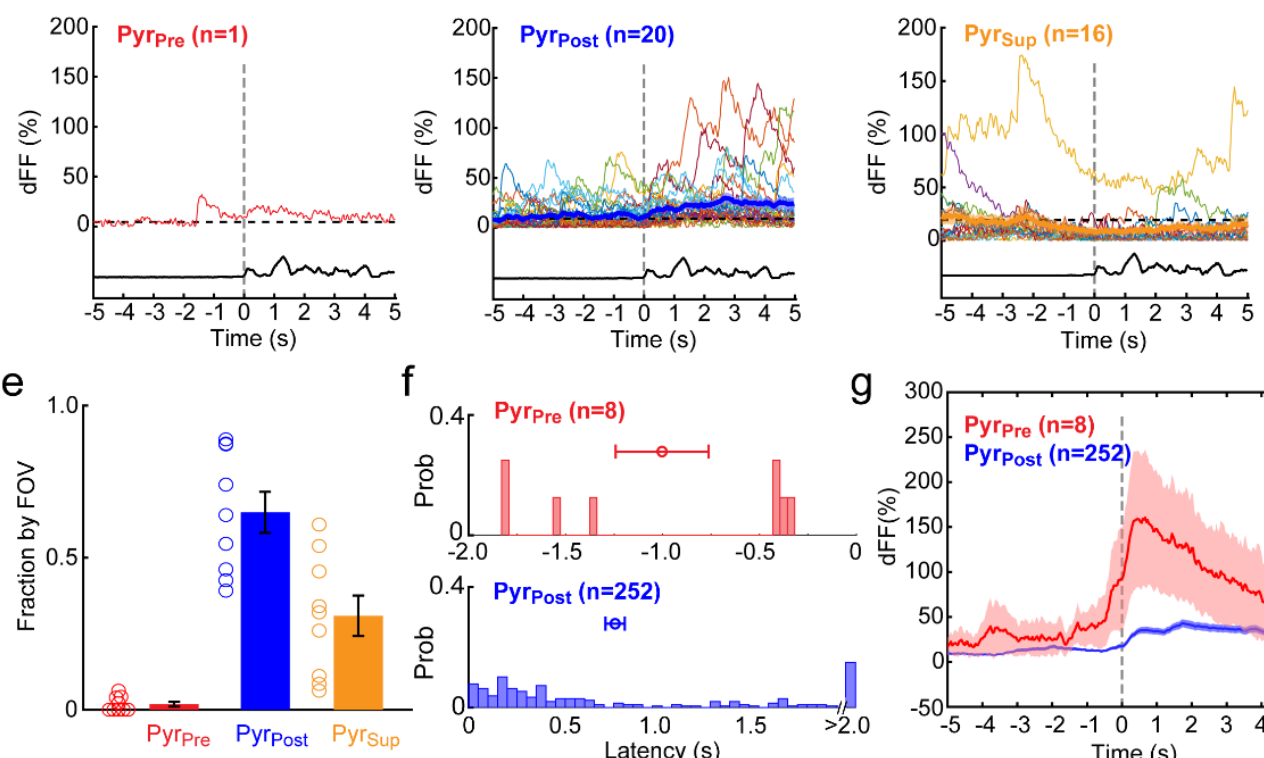

f
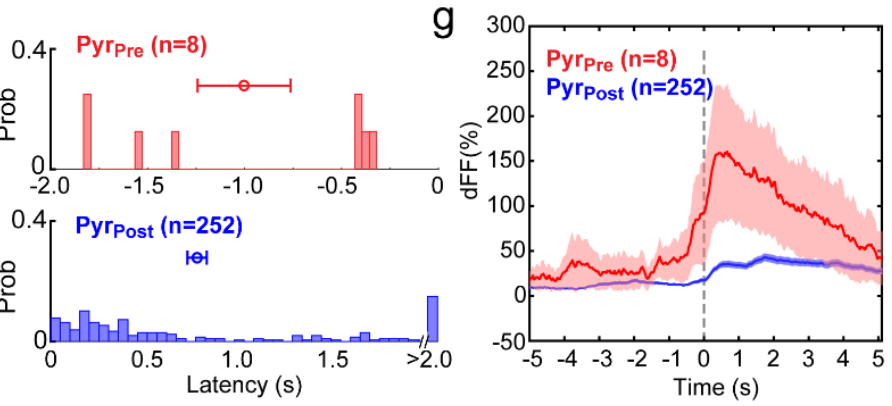

$\mathrm{h}$

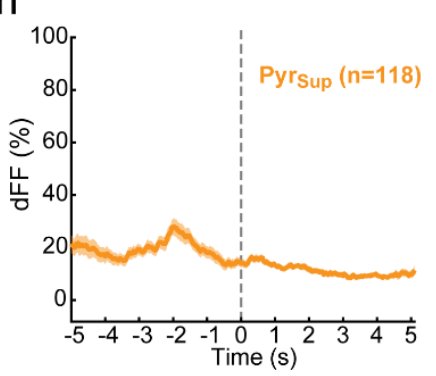

i

AAV-CAG-DIO-TVA-mCherry

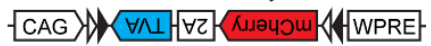
AAV-CAG-DIO-RG CAG WY OY WPRE

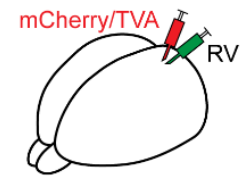

V1
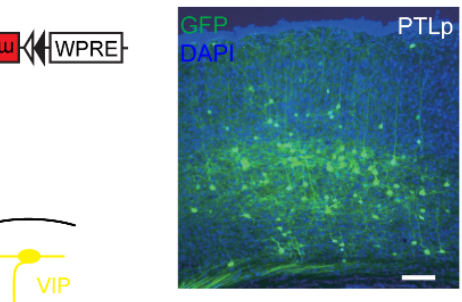

j

VIP/SST-Cre mice
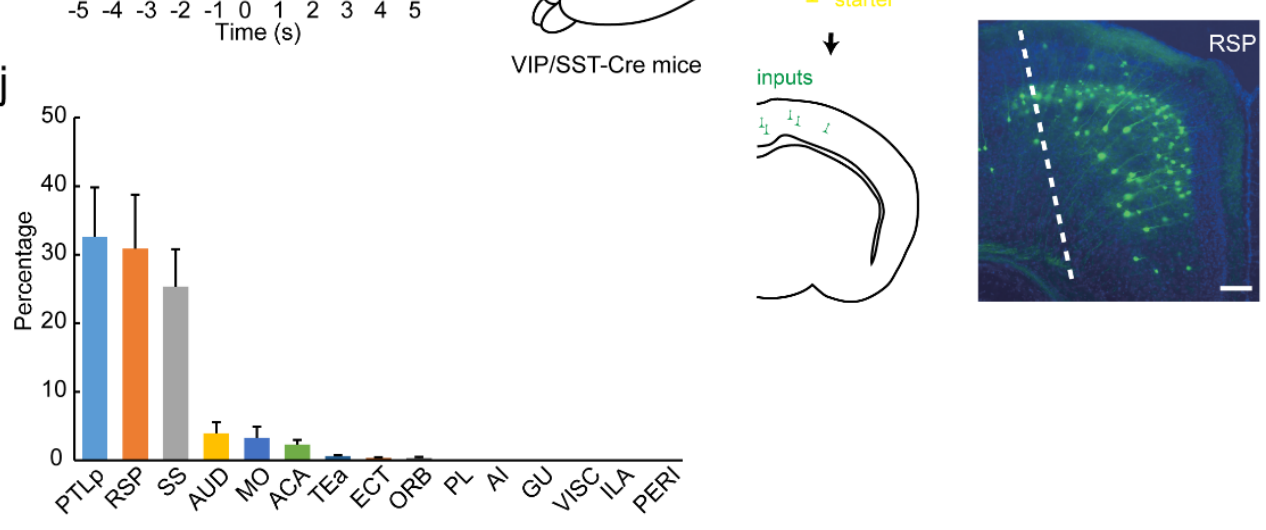
Figure 2. Distal top-down, but not local excitatory inputs, drive pre-running activation of VIP pre $_{\text {neurons. a. }}$ Example FOV from an Emx1-IRES-Cre;CaMKIla-tTA;Ai94 (GCaMP6s) mouse. Data display is similar to Fig. 1. b-d. Peri-running analysis of pyramidal cells in a. Activities of $\operatorname{Pyr}_{\text {Pre }}(\mathbf{b}), \operatorname{Pyr}_{\text {post }}(\mathbf{c})$ and $\operatorname{Pyr}_{\text {sup }}$ cells (d) are plotted. Note only 1 (2\%) Pyr Pre $_{\text {peuron among }} 47$ Pyr cells imaged. e - h. Population data showing proportions of Pyr Pre, Pyr $_{\text {Post }}$ and Pyr $_{\text {Sup }}$ neurons per FOV (e); latency distributions of Pyr $_{\text {pre }}$ (mean \pm SEM: $-1.000 \pm 0.240$ s) and Pyr Post $_{\text {( }}(0.758 \pm$ 0.046 s) cells (f); average time courses of $\operatorname{Pyr}_{\text {Pre, }} \operatorname{Pyr}_{\text {post }}(\mathbf{g})$ and $\operatorname{Pyr}_{\text {sup }}$ populations (h). The scarcity of $\operatorname{Pyr}_{\text {Pre }}$ neurons across all FOVs, which proves the pre-running activation of VIP INs is not running or data analysis artifacts, strongly suggests local inputs are insufficient to drive pre-running VIP activation. i\&j. VIP INs receive direct mono-synaptic top-down excitatory projections from frontal, parietal and retrosplenial motion-planning brain regions. i. Left: Schema of retrograde tracing of pre-synaptic inputs of V1 VIP neurons with engineered Cre-dependent rabies virus. Right: Example coronal section images showing dense fluorescently labeled input neurons in lateral posterior parietal cortex (PTLp, top) and retrosplenial cortex (RSP, bottom), respectively. Scale bar: $100 \mu \mathrm{m}$. j. Quantification of neocortical monosynaptic inputs to V1 L2/3 VIP neurons by cortical regions. Extrastriatal inputs to VIP neurons are dominated by PTLp, RSP and Somatosensory (SS) cortices, which are known to fire preparatory activities before voluntary actions. Brain regions are ranked by their relative input strength, from high to low. AUD: Auditory cortex; MO: Motor cortex; ACA: Anterior Cingulate cortex; TEa: Temporal association areas; ECT: Ectorhinal area; ORB: Orbital area; PL: Prelimbic area; Al: Agranular insular area; GU: Gustatory areas; VISC: Visceral area; ILA: Infralimbic area; PERI: Perirhinal area. Error bars: s.e.m. 
bioRxiv preprint doi: https://doi.org/10.1101/2021.12.31.474667; this version posted January 2, 2022. The copyright holder for this preprint (which was not certified by peer review) is the author/funder, who has granted bioRxiv a license to display the preprint in perpetuity. It is made available under aCC-BY-NC-ND 4.0 International license.

a

SST-IRES-Cre;Ai163
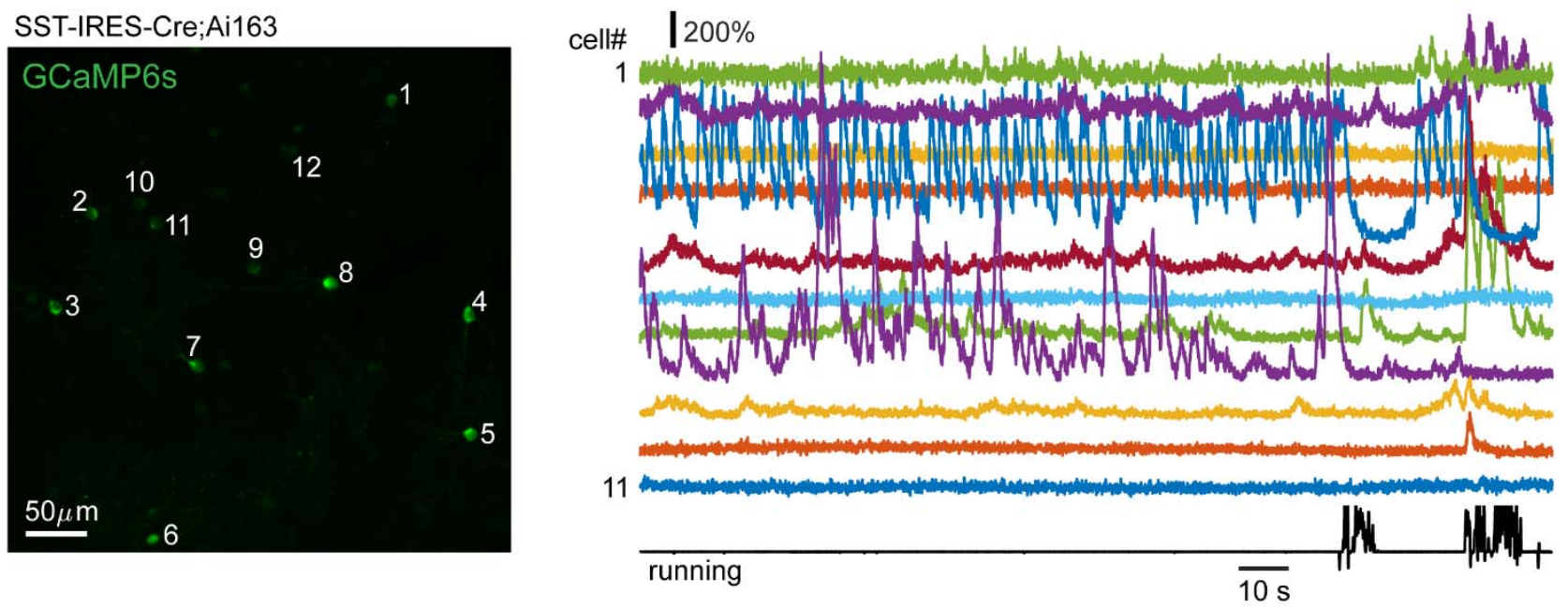

b

C
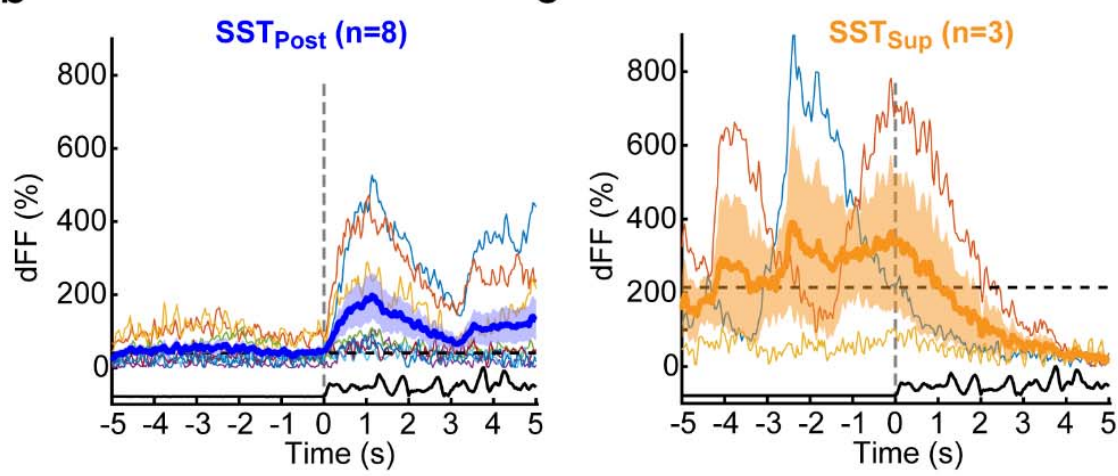

d

e

f
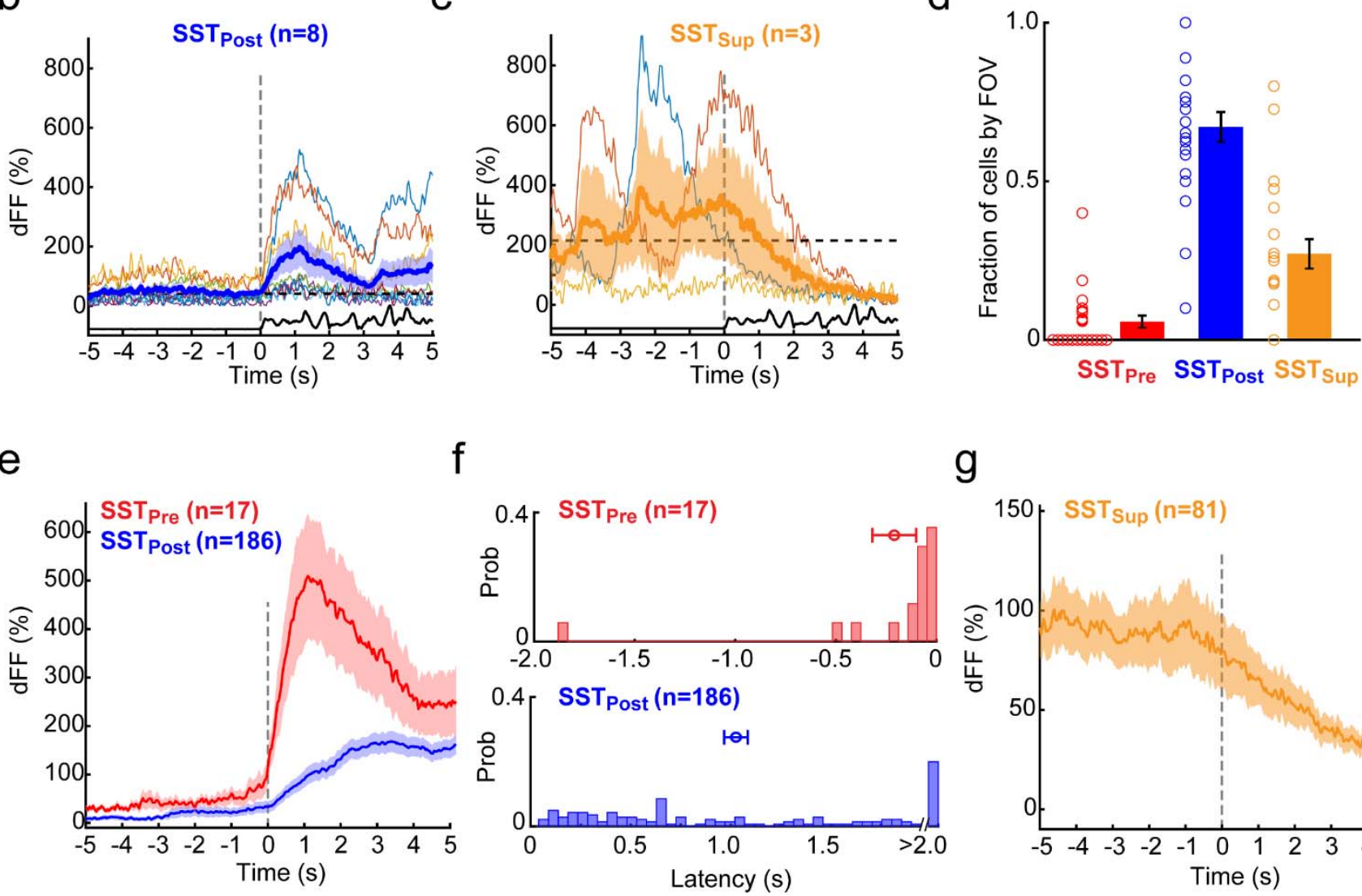

9

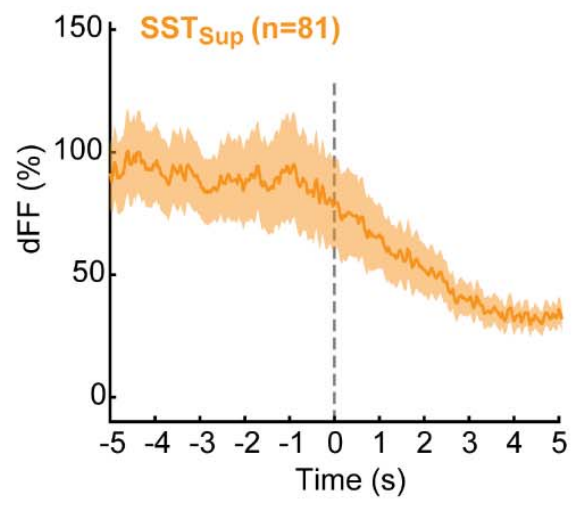


Figure 3. VIP-SST motif is a key circuit component of visual predictive processing. a. Example FOV in an SSTCre;Ai163 (GCaMP6s) mouse. $\mathbf{b}$ - d. Peri-running analysis of SST neurons in a. Activities of 8 SST post $(\mathbf{b})$ and 3

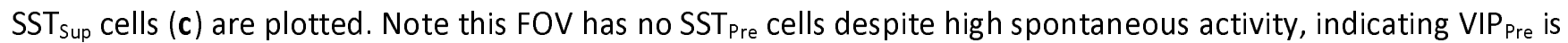
NOT caused by coincidental spontaneous firing or undocumented movement. e-g. Population data showing profound pre-running suppression and post-running activation in SST neurons. Individual panels show fractions of $\mathrm{SST}_{\text {pre, }} \mathrm{SST}_{\text {post }}$ and SST $\mathrm{Sup}_{\text {sup }}$ neurons by FOV (e); latency distributions of $\mathrm{SST}_{\text {pre }}$ (mean \pm s.e.m: $-1.000 \pm 0.240 \mathrm{sec}$ ), $\mathrm{SST}_{\text {Post }}(0.758 \pm 0.046 \mathrm{sec})$ cells, respectively $(\mathbf{f})$; average time courses of $\operatorname{Pyr}_{\text {Pre, }}, \operatorname{Pyr}_{\text {post }}(\mathbf{g})$ and $\mathrm{Pyr}_{\text {Sup }}$ populations (h). Note that high spontaneous firing does not necessarily result in robust and strong pre-running activation like that in VIP INs. Furthermore, unlike VIP Pre, SST $_{\text {pre }}$ neurons typically have strong post-running activation, which overwhelms the pre-running activities. Error bars: s.e.m. 
a

Emx1-IRES-Cre;CaMK2a;Ai94

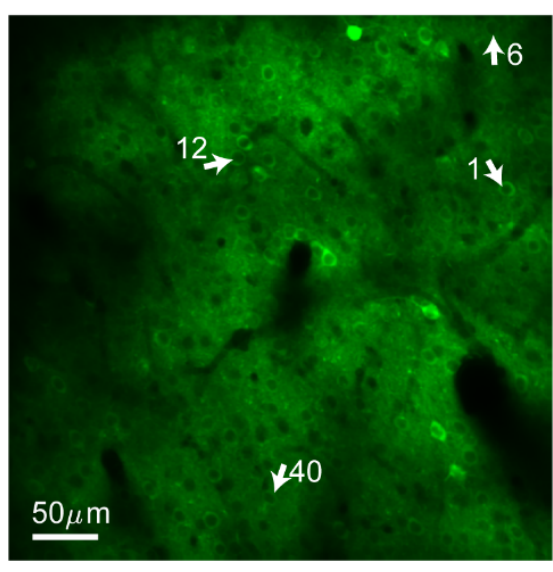

b

running + grey screen
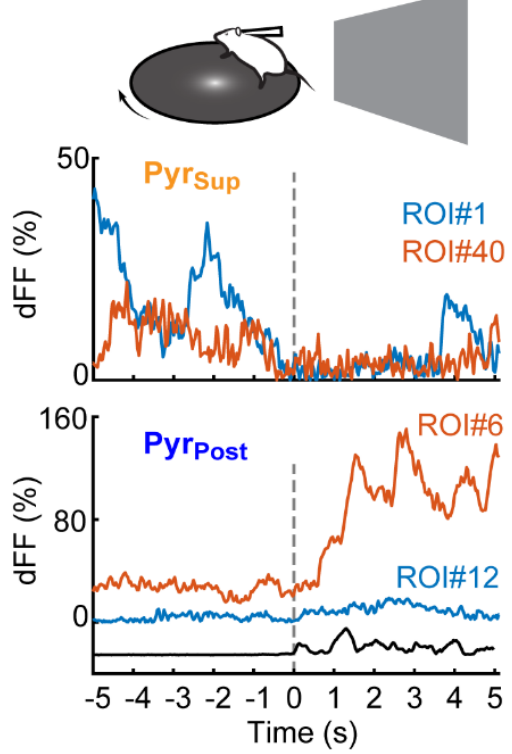

drifting gratings
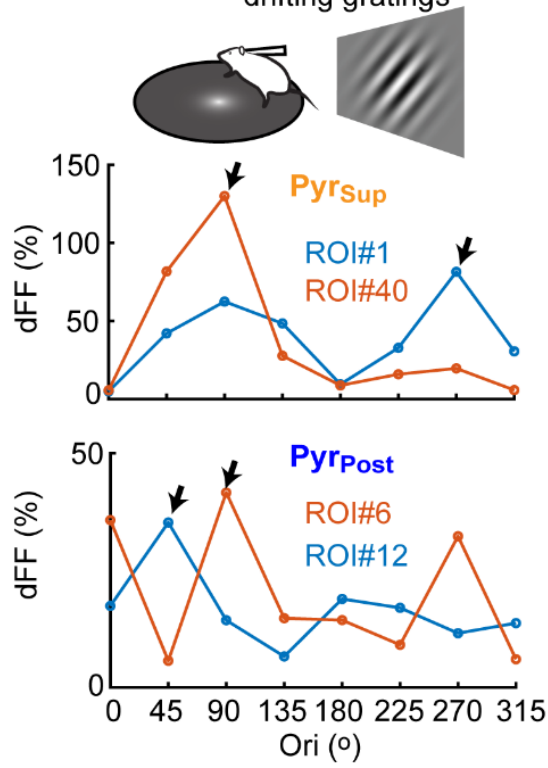

C

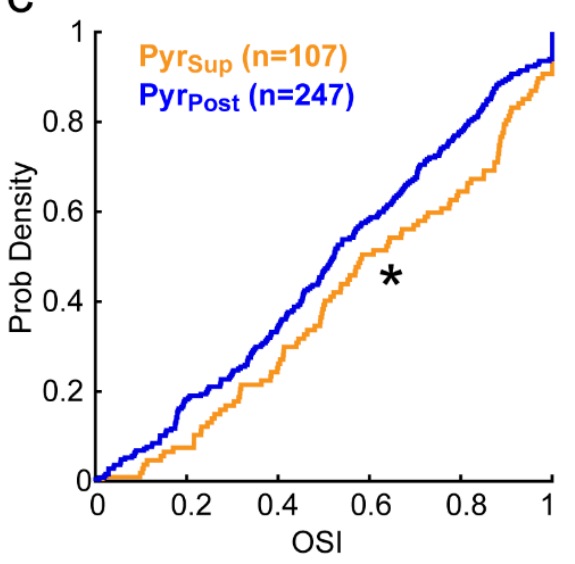

e

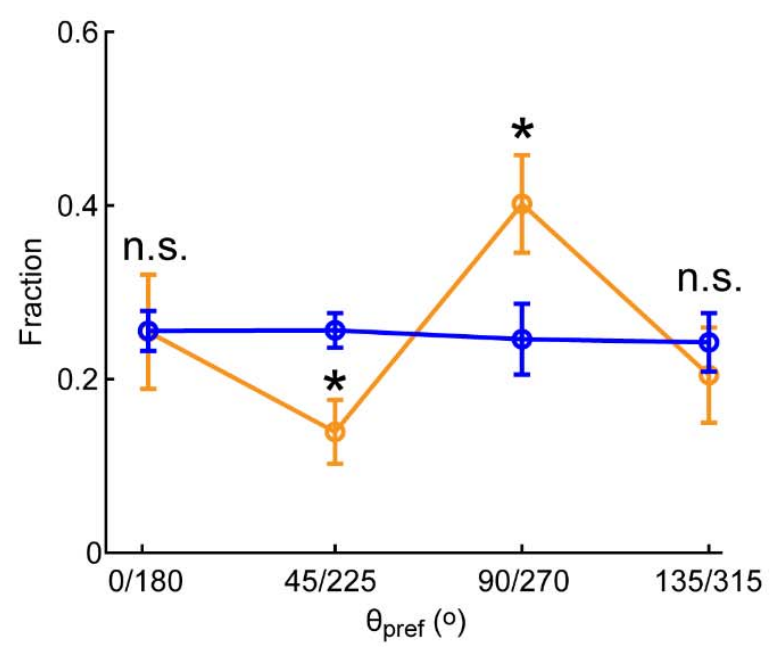

d
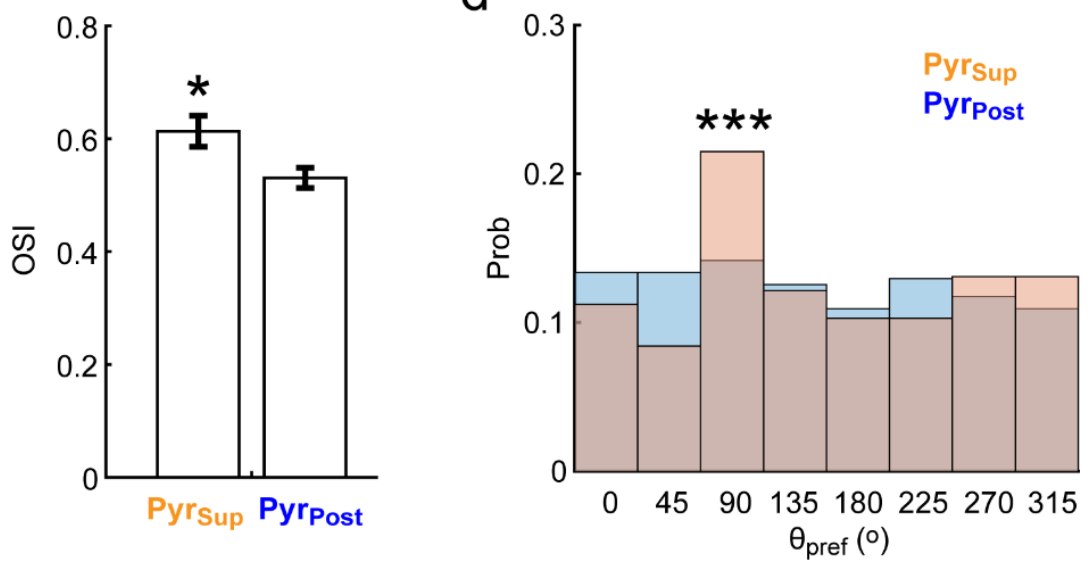

f

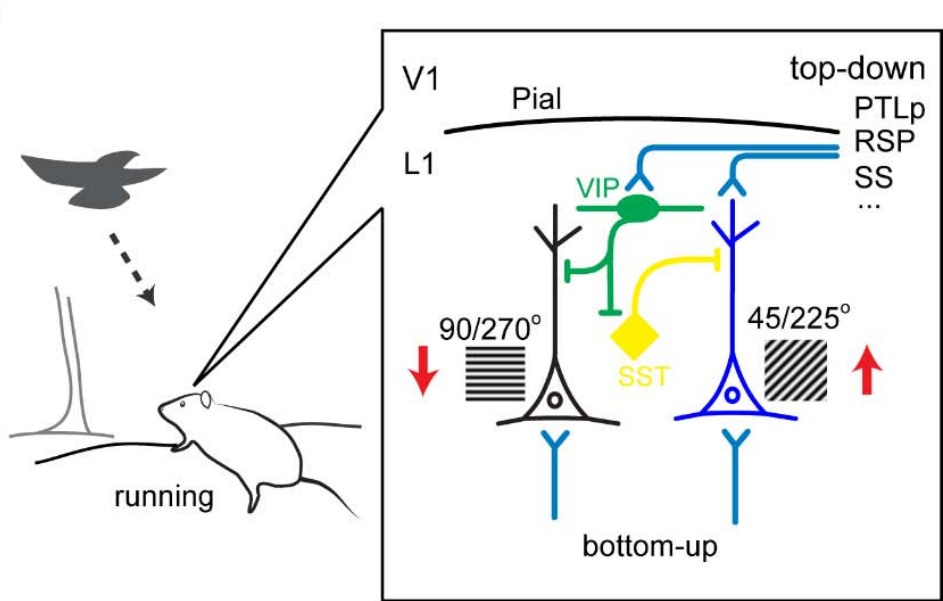


Figure 4. Adaptive modulation of Pyr orientation tuning by predictive processing during voluntary running.

a\&b. Orientation tuning curve mapping in the same set of Pyr neurons characterized during voluntary running. a. Z-projection image of the same set of Pyr cells in Fig. 2a. b. Orientation tuning mapping of identified Pyr $r_{\text {Sup }}$ and Pyr $_{\text {post }}$ cells (left column) with whole-screen drifting gratings (right column). Data are from corresponding ROls labeled in a. Black arrows indicate the preferred orientation $\left(\theta_{\text {pref }}\right)$. c. $\mathrm{Pyr}_{\text {sup }}$ and $\mathrm{Pyr}_{\text {post }}$ neurons significantly differ in orientation selectivity, as quantified by differences in cumulative distributions (left panel) and mean values (right panel) of OSI between $\mathrm{Pyr}_{\text {sup }}$ and Pyr $\mathrm{P}_{\text {post }}$ cells. d. $\theta_{\text {pref }}$ distributions of $\mathrm{Pyr}_{\text {sup }}$ and Pyr $\mathrm{P}_{\text {post }}$ neurons. Note $\theta_{\text {pref }}$ in $\mathrm{Pyr}_{\text {post }}$ nearly follows a uniform distribution, but deviates considerably from it in $\mathrm{Pyr}_{\text {sup }}$ cells, especially at $45^{\circ}$ and $90^{\circ}$. e. Normalization of $\theta_{\text {pref }}$ distributions by FOVs confirms that voluntary running anisotropically modulates $\mathrm{Pyr}_{\text {sup }}$ neurons, but the post-running excitation is isotropic. f. Peri-running neural dynamics in the VIPSST-Pyr microcircuit may help the brain adapt to incoming behaviorally salient sensory features (see main text for detail). ${ }^{*}: p<0.05, * * *: p<0.001$, n.s.: not significant, Error bars: s.e.m. 


\section{Methods}

Experimental procedures were in accordance with NIH guidelines and approved by the Institutional Animal Care and Use Committees (IACUC) of Sun Yat-sen University, Shanghai Jiao Tong University School of Medicine and Allen Institute for Brain Science. In the current study, 2-p Ca imaging were performed in adult transgenic mice ( 2 - 7 months, both sex), including VIP-IRES-Cre;Ai162 (GCaMP6s, n = 6), Emx1-IRES-Cre;CaMK2a-tTA;Ai94 (GCaMP6s, $n=3$ ) and SST-IRES-Cre;Ai163 (GCaMP6s, $n=5$ ). Monosynaptic retrograde tracing was conducted with VIP-IRES-Cre mice $(n=4)$. Optogenetic manipulation was done with VIP-IRES-Cre;Ai39 mice (Halo, $n=9)$. Viral injections. Viral vectors were prepared as published previously ${ }^{36,39}$. For retrograde monosynaptic tracing Glycoprotein-deleted $(\Delta G)$ and EnvA-pseudotyped rabies virus (RV- $\triangle \mathrm{G}-\mathrm{EGFP}+\mathrm{EnvA}, 2.4 \times 10^{8} \mathrm{IU} / \mathrm{mL}$ ), AAV2-CAGDIO-TVA-mCherry $\left(2.1 \times 10^{13} \mathrm{gc} / \mathrm{mL}\right)$ and AAV2-CAG-DIO-Glycoprotein $\left(8.7 \times 10^{12} \mathrm{gc} / \mathrm{mL}\right)$ were used. The genomic titer of AAVs and RV was determined by quantitative polymerase chain reactions. Both AAVs and RV were produced by BrainVTA. In order to trace presynaptic inputs of VIP neurons in V1, AAV2-CAG-DIO-TVAmCherry and AAV2-CAG-DIO-Glycoprotein (1:2, $500 \mathrm{~nL}$ ) were co-injected into V1 $(3 \mathrm{~mm}$ posterior and $2.5 \mathrm{~mm}$ lateral to Bregma, depth $0.5 \mathrm{~mm}$ ) of VIP-Cre mice. This resulted in cell-type specific expressions of TVA receptor and rabies glycoprotein, which are required for virus infection and trans-synaptic spread, respectively, in Crepositive VIP neurons. Two to three weeks later, RV- $\triangle \mathrm{G}-\mathrm{EGFP}+\mathrm{EnvA}(500 \mathrm{~nL}$ ) was injected into the same site as AAV injection. Histology was conducted 7 days after rabies virus injection. For optogenetics, AAV2-CaMKIIhChR2(H134R)-EYFP (UNC Vector Core) and AAV-DJ-CaMKII-hChR2(H134R)-EYFP (Stanford Neuroscience Gene Vector and Virus Core) were used. To activate top-down projections in V1, AAV2-hChR2(H134R) or AAV-DJhChR2(H134R) was injected into ipsilateral frontal cortex ( $800 \mathrm{~nL}, 0.2 \mathrm{~mm}$ anterior and $0.3 \mathrm{~mm}$ lateral to Bregma, depth $0.9 \mathrm{~mm}$ ).

Surgery. Detailed surgical procedures have been published elsewhere ${ }^{35}$. Briefly, a custom-made, pre-notched metal head-post was implanted under Isoflurane anesthesia (1.5 - 2\% in $\mathrm{O}_{2}$ ). A circular craniotomy was performed over left visual cortical area followed by durotomy. Silastic sealant (Kwik Sil) was applied to the craniotomy before a $3 \mathrm{~mm} \times 3 \mathrm{~mm}$ polycarbonate coverslip was placed over the exposed cortical area centering on $1.3 \mathrm{~mm}$ anterior and $3.1 \mathrm{~mm}$ lateral to the Lambda. Notches on the head-post were carefully aligned during surgery to establish a coordinate system for V1 positioning in Ca imaging. The headpost and coverslip were cemented firmly onto the skull. After 7 days of recovery, mice were habituated to head fixation and presentation of visual stimulations on an in-house made running device (a freely-rotating running disc) 2 hours per day for 1 week before imaging experiments started. During the daily habituation, mice were free to rest or run on the running disc, but not specifically trained for continuous locomotion. 
In vivo 2-p Ca Imaging. Ca imaging was performed using a Bruker 2-p microscope equipped with 8 kHz resonant scanners and a Chameleon Ultra II Ti:Sapphire femtosecond laser system (Coherent). The microscope was within a light-proof Faraday cage, which sufficiently blocked ambient light from surrounding laboratory area when the front doors were shut. Fluorescence was excited at $920 \mathrm{~nm}$, collected into two spectral channels using green $(510 / 42 \mathrm{~nm})$ and red (641/75 nm) emission filters and saved on a local computer. Imaging was conducted $150-$ $300 \mu \mathrm{m}$ underneath the pial surface with a 16x water-immersion objective lens (Nikon, NA 0.8). Image data were acquired at $512 \times 512$ pixels, $30 \mathrm{~Hz}$ frame rate with laser power $<70 \mathrm{~mW}$ measured after objective to ensure image quality. During imaging, mice were awake, head-fixed and allowed to run or rest on the running disc 'at will' (Fig. S1b), with or without visual stimulations. For imaging in darkness, in addition to turning off the visual stimulation monitor and covering all light emission spots within the Faraday cage, a custom-made black light shield, which fitted tightly with the headpost, was installed before imaging to fully cover the objective.

In a typical imaging experiment, we first imaged Ca activities within the $400 \times 400 \mu \mathrm{m}$ field of view (FOV) independent of running occurrence for $200 \mathrm{sec}$ without presentation of structured visual stimuli. A grey screen was presented during imaging on a calibrated LCD screen at $50 \%$ contrast to the contralateral eye, unless imaging was conducted in darkness. Orientation tuning mapping might follow after a $5-10$ minute break and was conducted to the same FOV with drifting grating stimuli (see below). Vasculature landmarks and coordinates calculated from headpost notches were used to keep FOVs from overlapping. To ensure animal's health, total imaging time in the same animal on the same day was less than 2 hours.

In vivo cell-attached recording. Mice were anesthetized with urethane $(0.6-1.2 \mathrm{~g} / \mathrm{kg}, \mathrm{IP})$ and chlorprothixene (5 $\mathrm{mg} / \mathrm{kg}, \mathrm{IM}$ ) after at least 2 weeks of ChR2 expression. During experiment, body temperature of the animal was maintained at $37^{\circ} \mathrm{C}$ using a feedback-controlled heating pad. A craniotomy was made over V1 after firmly cementing a custom head-post onto the skull. V1 Pyr neurons were recorded with borosilicate micropipettes ( 4-8 MS) filled with HEPES buffered ACSF under cell-attached configuration. Signals were acquired with a Multiclamp 700B amplifier, filtered between $0.5-2 \mathrm{kHz}$, sampled at $10 \mathrm{kHz}$ and stored on a local PC using the Clampex software. ACSF was frequently applied to the craniotomy to prevent the exposed cortex from drying.

Visual stimulations. Whole-screen sinusoidal drifting gratings were generated using Psychopy software and presented on the calibrated LCD monitor. The centre of the monitor was positioned $\sim 22 \mathrm{~cm}$ away from the centre of the contralateral eye. The stimulus set consisted of 8 orientations $\left(0^{\circ}-315^{\circ}, 45^{\circ}\right.$ increment), 4 spatial frequency (SF, $[0.02,0.04,0.08,0.16]$ cycle per degree, $c p d)$ and 1 temporal frequency $(2 \mathrm{~Hz})$. Gratings were presented to the animal at $80 \%$ contrast for 2 seconds, separated by 2 seconds of grey screen at mean illuminance and repeated 5 times in a random sequence. For optogenetics experiments, drifting gratings were 
presented for $4 \mathrm{sec}$. A grey screen at mean illuminance was randomly inserted and presented for 15 times. Due to the choice of whole-screen visual stimuli, we didn't map the receptive field or tract the pupil position.

Optogenetics. ChR2-expressing axons of frontal top-down projections were focally activated using blue laser pulses (wavelength $473 \mathrm{~nm}, 10 \mathrm{~Hz}, 5 \mathrm{~ms}$ ) delivered via an optic fiber (200 $\mu \mathrm{m}$ in diameter) over V1. The power of laser was measured at $\sim 3-5 \mathrm{~mW}$ at the open end of the optic fiber. To inactivate Halo-expressing V1 VIP INs, an optic-fiber (600 $\mu \mathrm{m}$ in diameter) was used to deliver yellow laser (wavelength $593 \mathrm{~nm}, 3 \mathrm{sec}$, power of 5-8 mW) over V1. Pyr responses to whole-screen drifting gratings were compared between no laser (ctrl), blue laser (topdown+) and blue+yellow lasers (top-down+/VIP-) conditions, representing control, top-down activation (resembling running) and inactivation of V1 VIP INs during running, respectively. The open ends of optic fibers were held in place over the recorded Pyr neurons and in close proximity to the exposed V1 surface by micromanipulators.

Histology. Detail of histology has been published elsewhere ${ }^{36}$. After embedding and freezing, the brain was sectioned into 50- $\mu \mathrm{m}$ coronal slices using a cryostat. One out of every three sections were imaged in the highthroughput slide scanners (Nanozoomer-2.0RS) for further processing. A custom written software package was used to analyze the digitized brain images. The analysis software consists of four modules: atlas rotation, image registration, signal detection, and quantification/visualization. The Allen Mouse Brain Atlas was rotated to mimic the aberrant sectioning angle of the experimental brain. Images of brain sections were aligned to the rotated reference atlas for further quantification. The detection module was used to record the position of manually identified EGFP-labeled neurons in each digitized brain section image. After detection and registration, signals were quantified across the whole brain. Since the number of labeled neurons varied across brains, the input density from each region was quantified by dividing the number of labeled neurons found in that region by the total number of labeled neurons detected in the entire brain, with the exception of the injection site.

Data analysis. Behaviours of head-fixed mice during Ca imaging were quantified to validate the behavioural paradigm employed in the current study was adequate to study neural correlates of self-initiated (voluntary) actions. Data of running velocity were analyzed and results were summarized in Supplementary Figs. S1b, S7b and S9b, respectively. Firstly, in our experiments running was sporadic, i.e. mice didn't necessarily run in every imaging session. Secondly running was typically brief, only occurred in $\sim 5 \%-10 \%$ of the total imaging time. This distinguished the current study from those that specifically trained animals to run continuously, which engaged the neuromodulatory system profoundly. Thirdly, the start of running was random in time, which meant animals could initiate running at any time unknown to the experiment, in a way similar to the 'at will' performance in human studies. This was because running onset followed a uniform distribution, which made it impossible to deliver temporally coupled manipulation, and at the same time, directly argued against the possibility that the 
running represented a learned behaviour in response to head-fixation or was driven by external sensory cue. Finally, in our dataset, running exhibited these features independent of transgenic mouse lines or imaging conditions. We want to emphasize that our experiment design, especially the use of naive mice in absence of external sensory stimuli, ensures minimal engagement of selective attention and associative learning processes. Therefore, we concluded that the running behaviour in our paradigm was self-initiated and peri-running neuronal dynamics accurately reflected neural processes underlying voluntary running.

Ca imaging data were analyzed using in-house Matlab scripts. Green image stacks were first corrected for inplane motion artifacts using published cross-correlation motion correction method between frames ${ }^{54}$. Image segmentation was done with custom-made Matlab package ${ }^{34}$. Regular ring-shape ROls were defined and neuropil subtraction was conducted for Pyr cells imaged from Emx1-IRES-Cre;CaMK2a-tTA;Ai94 mice as described previously ${ }^{34}$. For inhibitory VIP and SST interneurons, as we reported before, GCaMP6s expression in VIP-IRES-Cre;Ai162 and SST-IRES-Cre;Ai163 mice showed no clear nucleus exclusion ${ }^{33}$, patch ROIs were thus used for VIP and SST interneurons and green fluorescence signals were computed from all pixels within the patch $\mathrm{ROI}^{34}$. Due to the sparseness of cortical inhibitory interneurons, neuropil signal was not corrected for VIP and SST neurons ${ }^{34}$.

For imaging data of voluntary running, $\Delta F / F$ was calculated for each $R O I$ with the mode of raw $F$ traces $F_{\text {mod, }}$ i.e. $\Delta F / F=\left(F-F_{\text {mod }}\right) / F_{\text {mod }}$. Fluorescently active ROls were then determined by threshoulding the $\Delta F / F$ traces at 34 standard deviations above the mean with manual verifications. Inactive ROIs were rejected from further analysis. To analyze peri-running neuronal dynamics, running velocity were threshoulded at $1 \mathrm{~cm} / \mathrm{s}$ (ref \#44). The time points at which the velocity up- and down-crossed the $1 \mathrm{~cm} / \mathrm{s}$ threshould defined running onsets and offsets, respectively. Consistent with previous results ${ }^{42,44}, 1 \mathrm{~cm} / \mathrm{s}$ is at the low end of the velocity distribution. For better temporal resolution, velocity data were not smoothed. Imaging sessions that failed to reach the velocity threshould were considered no running and excluded from further analysis. Next for each imaging session, we identified running episodes during which the mice remained stationary (i.e. no suprathreshould events detected) for at least $10 \mathrm{sec}$ before the running onset, in order to control the slow decay of GCaMP6s. $\triangle F / F$ traces of active ROls within -10 s to $5 \mathrm{~s}$ from the running onset were extracted (using the imaging frame nearest to the running onset as time 0) and averaged across running episodes, in order to control the variabilities between running episodes. The averaged $\Delta F / F$ traces were carefully examined with the running data for suspicious pre-running subthreshould movement. Data showing $\Delta \mathrm{F} / \mathrm{F}$ deflection corresponding with the subthreshould movement were excluded from analysis, unless could be proved otherwise (e.g. Fig. 1c\&d). For individual ROI, latency was determined from running-episode averaged $\Delta \mathrm{F} / \mathrm{F}$ trace as the earliest time point (in the [-3s, 2s] range) above the mean plus 3 - 4 times standard deviation, both computed from the [-10s, 0s] baseline data. In a small 
number of cells $(<5 \%)$, baseline was slightly adjusted, e.g. to $[-10 s,-1 s]$ or $[-10 s,-2 s]$, to deal with the excessive firing just before the running initiation. Cells with negative and positive latencies were classified as 'Pre' and 'Post' cells, respectively. Those cells that failed to meet the latency criteria but had mean $\Delta \mathrm{F} / \mathrm{F}$ within [0s, 5s] lower than mean baseline $\Delta F / F$ were categorized into the suppressed-by-running group ('Sup'). The rest was considered non-responsive cells. We found this resulted in complete, non-overlapping classification of VIP, SST, Pyr neurons. To control the variation of post-running activities among individual neurons, $\Delta F / F$ was normalized by the group average of $\Delta F / F$ during corresponding baseline activities. We also normalized $\Delta F / F$ individually by the average of baseline activity, which controlled baseline variations, and results were consistent (data not shown). Due to the slow decay of GCaMP6s, we didn't analyze $\triangle F / F$ activities for running offsets.

To analyze visually evoked $C$ a responses, $\Delta F / F$ was calculated for individual trials. The average $F$ signal within $2 \mathrm{sec}$ immediately before the stimulus onset were used as the baseline. In case that companion imaging sessions of voluntary running were available, ROls were imported from the corresponding running session, adjusted for any spatial shifts to ensure best alignment. For visual response analysis, fluorescently active ROls were analyzed independent of the occurrence of running. $\Delta \mathrm{F} / \mathrm{F}$ was averaged over the $2 \mathrm{sec}$ stimulus duration, then by stimulus conditions. To calculate the orientation selectivity index (OSI), the preferred orientation $\left(\theta_{\text {pref }}\right)$ was determined by the orientation that evoked the strongest response at the preferred SF. OSI was calculated as OSI $=\left(R_{\text {pref }}-\right.$ $\left.R_{\text {orth }}\right) /\left(R_{\text {pref }}+R_{\text {orth }}\right)$, where $R_{\text {pref }}$ and $R_{\text {orth }}$ stands for the response magnitude at $\theta_{\text {pref }}$ and the orthogonal orientation $\left(\theta_{\text {pref }}+\pi / 2\right)$, respectively. Direction selectivity index $(D S I)$ was calculated as $D S I=\left(R_{\text {pref }}-R_{o p p o}\right) /\left(R_{\text {pref }}+R_{o p p o}\right)$, where $R_{\text {oppo }}$ stands for the response magnitude at the opposite direction $\left(\theta_{\text {pref }}+\pi\right)$.

Cell-attached data were analyzed using in-house scripts. Only recordings stable for more than 5 min and with sufficiently high seal resistance (10-100 $\mathrm{M} \Omega$ ) were included in final data analysis.

It has been reported that some Emx1-IRES-Cre;CaMK2a-tTA;Ai94 mice could have aberrant epileptiform activity, which may confound our Pyr data. No signs of seizure-like behavior were noticed either during imaging or in home-cages from the Ai94 mice included in our data analysis. Running statistics of these Ai94 mice showed no difference from those VIP and SST transgenic mice we imaged, and Pyr responsiveness was fully comparable with that previously reported in normal mice. All these pieces of evidence suggested no behavioral abnormality in these three Ai94 mice, thus our Ai94 results were NOT confounded by epileptiform activity that may occur in some Ai94 mice. 


\section{References}

${ }^{1} \mathrm{H}$. von Helmholtz. Concerning the perceptions in general (3rd edn.), Treatise on Physiological Optics, Vol. III (1866) (translated by J. P. C. Southall 1925)

${ }^{2}$ Wolpert DM, Ghahramani Z, Jordan MI. An internal model for sensorimotor integration. Science 269(5232):1880-2 (1995). doi: 10.1126/science.7569931.

${ }^{3}$ Rao RP, Ballard DH. Predictive coding in the visual cortex: a functional interpretation of some extra-classical receptive-field effects. Nat Neurosci. 2(1):79-87 (1999). doi: 10.1038/4580.

${ }^{4}$ Körding KP, Wolpert DM. Bayesian integration in sensorimotor learning. Nature 427(6971):244-7 (2004). doi: 10.1038/nature02169.

${ }^{5}$ Friston K. The free-energy principle: a unified brain theory? Nat Rev Neurosci 11(2):127-38 (2010). doi: $10.1038 / \mathrm{nrn} 2787$.

${ }^{6}$ Clark A. Whatever next? Predictive brains, situated agents, and the future of cognitive science. Behav Brain Sci. 36(3):181-204 (2013). doi: 10.1017/S0140525X12000477.

${ }^{7}$ Bubic A, von Cramon DY, Schubotz RI. Prediction, cognition and the brain. Front Hum Neurosci 22;4:25 (2010). doi: 10.3389/fnhum.2010.00025.

${ }^{8}$ Franklin DW, Wolpert DM. Computational mechanisms of sensorimotor control. Neuron. 72(3):425-42 (2011). doi: 10.1016/j.neuron.2011.10.006.

${ }^{9}$ Brown EC, Brüne M. The role of prediction in social neuroscience. Front Hum Neurosci. 6:147 (2012). doi: 10.3389/fnhum.2012.00147.

${ }^{10}$ den Ouden HEM, Kok P, de Lange FP. How prediction errors shape perception, attention, and motivation. Front Psychol 11;3:548 (2012). doi: 10.3389/fpsyg.2012.00548

${ }^{11}$ Spratling MW. A review of predictive coding algorithms. Brain Cogn. (2017).

${ }^{12}$ Griffin JD, Fletcher PC. Predictive Processing, Source Monitoring, and Psychosis. Annu Rev Clin Psychol. 13:265-289 (2017). doi: 10.1146/annurev-clinpsy-032816-045145.

${ }^{13}$ Kornhuber, H.H. \& Deecke, L. Hirnpotentialänderungen bei Willkürbewegungen und passiven Bewegungen des Menschen: Bereitschaftspotential und reafferente Potentiale. Pflügers Arch. ges. Physiol. 284, 1-17 (1965).

${ }^{14}$ Libet, B., Gleason, C. A., Wright, E. W. \& Pearl, D. K. Time of conscious intention to act in relation to onset of cerebral activity (readiness potential). The unconscious initiation of a freely voluntary act. Brain 106, 623-642 (1983).

${ }^{15}$ Libet, B. Unconscious cerebral initiative and the role of conscious will in voluntary action. Behav. Brain Sci. 8, 529-566 (1985).

${ }^{16}$ Okano, K. \& Tanji, J. Neuronal activities in the primate motor fields of the agranular frontal cortex preceding visually triggered and self-paced movement. Exp. Brain Res. 66, 155-166 (1987). 
${ }^{17}$ Romo R. \& Schultz W. Role of primate basal ganglia and frontal cortex in the internal generation of movements. III. Neuronal activity in the supplementary motor area. Exp Brain Res. 91(3):396-407 (1992). doi: 10.1007/BF00227836.

${ }^{18}$ Fried I, Mukamel R, Kreiman G. Internally generated preactivation of single neurons in human medial frontal cortex predicts volition. Neuron. 69(3):548-62 (2011). doi: 10.1016/j.neuron.2010.11.045.

${ }^{19}$ Murakami M, Vicente MI, Costa GM, Mainen ZF. Neural antecedents of self-initiated actions in secondary motor cortex. Nat Neurosci. 17(11):1574-82 (2014). doi: 10.1038/nn.3826.

${ }^{20}$ Li N, Daie K, Svoboda K, Druckmann S. Robust neuronal dynamics in premotor cortex during motor planning. Nature. 532(7600):459-64 (2016). doi: 10.1038/nature17643.

${ }^{21}$ Eliades SJ, Wang X. Neural substrates of vocalization feedback monitoring in primate auditory cortex. Nature 453:1102-1106 (2008).

${ }^{22}$ Keller GB, Hahnloser RH. Neural processing of auditory feedback during vocal practice in a songbird. Nature 457:187-190 (2009).

${ }^{23}$ Keller GB, Bonhoeffer T, Hubener M. Sensorimotor mismatch signals in primary visual cortex of the behaving mouse. Neuron 74:809-815 (2012). DOI: https://doi.org/10.1016/j.neuron.2012.03.040.

${ }^{24}$ Ayaz A, Stauble A, Hamada M, Wulf MA, Saleem AB, Helmchen F. Layer-specific integration of locomotion and sensory information in mouse barrel cortex. Nature Communications 10:2585 (2019). DOI:

https://doi.org/10.1038/s41467-019-10564-8.

${ }^{25}$ Ramón y Cajal, S. Comparative study of the sensory areas of the human cortex. (1899)

${ }^{26}$ Markram H, Toledo-Rodriguez M, Wang Y, Gupta A, Silberberg G, Wu C. Interneurons of the neocortical inhibitory system. Nat Rev Neurosci. 5(10):793-807 (2004)

${ }^{27}$ Petilla Interneuron Nomenclature Group et al. Petilla terminology: nomenclature of features of GABAergic interneurons of the cerebral cortex. Nat Rev Neurosci. 9(7):557-68 (2008) doi: 10.1038/nrn2402.

${ }^{28}$ Kepecs A, Fishell G. Interneuron cell types are fit to function. Nature. 505(7483):318-26. (2014) doi: $10.1038 /$ nature12983.

${ }^{29}$ Keller GB \& Mrsic-Flogel TD. Predictive Processing: A Canonical Cortical Computation. Neuron. 100(2):424-435 (2018). doi: 10.1016/j.neuron.2018.10.003.

${ }^{30}$ Teufel C \& Fletcher PC. Forms of prediction in the nervous system. Nat Rev Neurosci. 21(5):297 (2020). doi: 10.1038/s41583-020-0296-0.

${ }^{31}$ Leinweber M et al. A Sensorimotor Circuit in Mouse Cortex for Visual Flow Predictions. Neuron. 96(5):1204 (2017). doi: 10.1016/j.neuron.2017.11.009.

${ }^{32}$ Madisen L, Zwingman TA, Sunkin SM, Oh SW, Zariwala HA, Gu H, Ng LL, Palmiter RD, Hawrylycz MJ, Jones AR, Lein $\mathrm{ES}$, Zeng $\mathrm{H}$. A robust and high-throughput Cre reporting and characterization system for the whole mouse brain. Nat Neurosci. 13(1):133-40. (2010) 
${ }^{33}$ Daigle, Madisen et al. A suite of transgenic driver and reporter mouse lines with enhanced brain cell type targeting and functionality. Cell, 12;174(2):465-480.e22 (2018). doi: 10.1016/j.cell.2018.06.035.

${ }^{34}$ Chen TW et al. Ultrasensitive fluorescent proteins for imaging neuronal activity. Nature 499(7458):295-300 (2013). doi: 10.1038/nature12354.

${ }^{35}$ Knoblich et al Neuronal cell-subtype specificity of neural synchronization in mouse primary visual cortex. Nat Commun. 10(1):2533. (2019) doi: 10.1038/s41467-019-10498-1.

${ }^{36} \mathrm{Ma} \mathrm{G}$ et al. Hierarchy in sensory processing reflected by innervation balance on cortical interneurons. Sci Adv. 7(20):eabf5676 (2021). doi: 10.1126/sciadv.abf5676.

${ }^{37}$ Lee $S$ et al. A disinhibitory circuit mediates motor integration in the somatosensory cortex. Nat Neurosci. 16(11):1662-70. (2013) doi: 10.1038/nn.3544. Epub 2013 Oct 6.

${ }^{38} \mathrm{Pi} \mathrm{HJ}$ et al. Cortical interneurons that specialize in disinhibitory control. Nature. 503(7477):521-4. (2013) doi: 10.1038/nature12676. Epub 2013 Oct 6.

${ }^{39}$ Zhang S. et al. Selective attention. Long-range and local circuits for top-down modulation of visual cortex processing. Science. 345(6197):660-5. (2014) doi: 10.1126/science.1254126.

${ }^{40}$ Ibrahim LA, Mesik L, Ji XY, Fang Q, Li HF, Li YT, Zingg B, Zhang LI, Tao HW. Cross-Modality Sharpening of Visual Cortical Processing through Layer-1-Mediated Inhibition and Disinhibition. Neuron. 89(5):1031-45. (2016) doi: 10.1016/j.neuron.2016.01.027. Epub 2016 Feb 18.

${ }^{41}$ Krabbe $S$ et al. Adaptive disinhibitory gating by VIP interneurons permits associative learning. Nat Neurosci. 22(11):1834-1843 (2019). doi: 10.1038/s41593-019-0508-y. Epub 2019 Oct 21.

${ }^{42}$ Fu Y, Tucciarone JM, Espinosa JS, Sheng N, Darcy DP, Nicoll RA, Huang ZJ, Stryker MP. A cortical circuit for gain control by behavioral state. Cell. 156(6):1139-52 (2014).

${ }^{43}$ Polack PO, Friedman J, Golshani P. Cellular mechanisms of brain state-dependent gain modulation in visual cortex. Nat Neurosci. 16(9):1331-9 (2013). doi: 10.1038/nn.3464. Epub 2013 Jul 21.

${ }^{44}$ Niell CM and Stryker MP. Modulation of visual responses by behavioral state in mouse visual cortex. Neuron 65(4):472-9. (2010) doi: 10.1016/j.neuron.2010.01.033.

${ }^{45}$ Rudy B, Fishell G, Lee S, and Hjerling-Leffler J. Three groups of interneurons account for nearly $100 \%$ of neocortical GABAergic neurons. Dev. Neurobiol. 71, 45-61 (2010) doi: 10.1002/dneu.20853.

${ }^{46}$ Pfeffer CK, Xue M, He M, Huang ZJ, Scanziani M. Inhibition of inhibition in visual cortex: The logic of connections between molecularly distinct interneurons. Nat. Neurosci. 16(8): 1068-1076 (2013)

${ }^{47}$ Attinger A., Wang B. and Keller GB. Visuomotor Coupling Shapes the Functional Development of Mouse Visual Cortex. Cell. 169(7):1291-1302.e14 (2017). doi: 10.1016/j.cell.2017.05.023.

${ }^{48}$ Lee SH, Kwan AC, Zhang S, Phoumthipphavong V, Flannery JG, Masmanidis SC, Taniguchi H, Huang ZJ, Zhang F, Boyden ES, Deisseroth K, Dan Y. Activation of specific interneurons improves V1 feature selectivity and visual perception. Nature. 488(7411):379-83. (2012) 
${ }^{49}$ Wilson NR, Runyan CA, Wang FL, Sur M. Division and subtraction by distinct cortical inhibitory networks in vivo. Nature. 488(7411):343-8. (2012)

${ }^{50}$ Atallah BV, Bruns W, Carandini M, Scanziani M. Parvalbumin-expressing interneurons linearly transform cortical responses to visual stimuli. Neuron. 73(1):159-70. (2012)

${ }^{51}$ Wang Q, Webber RM, Stanley GB. Thalamic synchrony and the adaptive gating of information flow to cortex. Nat. Neurosci. 13(12):1534-41. (2010) doi: 10.1038/nn.2670.

${ }^{52}$ Saxe A, Nelli S, Summerfield C. If deep learning is the answer, what is the question? Nat Rev Neurosci. 22(1):55-67 (2021). doi: 10.1038/s41583-020-00395-8.

${ }^{53}$ Haggard P. Human volition: towards a neuroscience of will. Nat Rev Neurosci. 9(12):934-46 (2008). doi: 10.1038/nrn2497.

${ }^{54}$ Dombeck DA, Khabbaz AN, Collman F, Adelman TL, Tank DW. (2007) Imaging large-scale neural activity with cellular resolution in awake, mobile mice. Neuron. 56(1):43-57. 